\title{
Crossing a Coupling Spin Resonance with an RF Dipole*
}

\author{
M. Bai, T. Roser \\ Brookhaven National Laboratory, Upton, NY 11973, U.S.A
}

\begin{abstract}
In accelerators, due to quadrupole roll errors and solenoid fields, the polarized proton acceleration often encounters coupling spin resonances. In the Brookhaven AGS, the coupling effect comes from the solenoid partial snake which is used to overcome imperfection resonances. The coupling spin resonance strength is proportional to the amount of coupling as well as the strength of the corresponding intrinsic spin resonance. The coupling resonance can cause substantial beam polarization loss if its corresponding intrinsic spin resonance is very strong. A new method of using an horizontal rf dipole to induce a full spin flip crossing both the intrinsic and its coupling spin resonances is studied in the Brookhaven's AGS. Numerical simulations show that a full spin flip can be induced after crossing the two resonances by using a horizontal rf dipole to induce a large vertical coherent oscillation.
\end{abstract}

\section{INTRODUCTION}

In an accelerator, particles undergo betatron oscillations in both horizontal and vertical planes while they circulate around the machine. In a perfect machine, both oscillations are independent of each other. However, this independence can be broken if there is any quadrupole roll errors or solenoid fields. In this case, the horizontal motion is coupled to the vertical oscillation. Unlike the uncoupled case, the frequency spectrum of the betatron oscillation in either of the two transverse plane then consists of two components $\nu_{1}$ and $\nu_{2}$ given by $[1,2]$

$$
\begin{array}{r}
\nu_{1}=\frac{1}{2}\left(\nu_{x}+\nu_{z}\right)+\frac{1}{2} \sqrt{\left(\nu_{x}-\nu_{z}\right)^{2}+\Delta Q_{\min }^{2}} \rightarrow \nu_{x} ; \\
\text { without coupling } \\
\nu_{2}=\frac{1}{2}\left(\nu_{x}+\nu_{z}\right)-\frac{1}{2} \sqrt{\left(\nu_{x}-\nu_{z}\right)^{2}+\Delta Q_{\text {min }}^{2}} \rightarrow \nu_{z} ; \\
\text { with couple }
\end{array}
$$

where $\nu_{x}$ and $\nu_{z}$ are the unperturbed horizontal and vertical tunes. $\Delta Q_{\min }$ is the minimum tune split between the two eigen tunes when $\nu_{x}=\nu_{z}$ and is proportional to the coupling strength [5]. With weak coupling,

$$
\begin{aligned}
\nu_{1} & \simeq \nu_{x} \\
\nu_{2} & \simeq \nu_{z} .
\end{aligned}
$$

* The work was performed under the auspices of the US Department of Energy
In the Brookhaven AGS, the main coupling source comes from the solenoid partial snake which is used to overcome the imperfection spin resonances in the AGS [3]. The minimum tune split $\Delta Q_{\min }$ from the $5 \%$ partial snake is about 0.015 .

In a coupled machine, in addition to the intrinsic spin resonance at $G \gamma=k P \pm \nu_{2}\left(G \gamma=k P \pm \nu_{z}\right.$ without coupling) [4], the vertical betatron oscillation also drives a coupling spin resonances at $G \gamma=k P \pm \nu_{1}$ [5]. The strength of the coupling resonance $\epsilon_{\nu_{x}}$ is proportional to the amount of the coupling and it is given by

$$
\epsilon_{\nu_{x}} \propto C_{x} \sqrt{\varepsilon_{u}} \epsilon_{\nu_{z}}
$$

where $\epsilon_{\nu_{z}}$ is the strength of the adjacent intrinsic spin resonance and $C_{x}$ is the coupling coefficient. For a fully coupled machine, $\nu_{x}=\nu_{z}$ and $C_{x}=1$. For a decoupled machine, $C_{x}=0 . \varepsilon_{u}$ is the beam emittance in the eigen direction [6] and equals the horizontal beam emittance if $C_{x}=0$.

In the AGS, there are four strong intrinsic spin resonances at $0+\nu_{z}, 12+\nu_{z}$ and $36 \pm \nu_{z}$ [4]. Traditionally in the AGS, the beam polarization loss at the coupling resonances is minimized by separating the horizontal and vertical tunes. The coupling resonances around these four strong intrinsic resonances can produce about $35 \%$ polarization losses with the normal AGS polarized proton setting $[7,8]$. In order to achieve $70 \%$ polarization in the AGS, one needs to minimize the polarization loss at the coupling resonances. Since they are adjacent to the intrinsic resonances, it is very difficult to use the vertical rf dipole [9] to obtain full spin flips at both the intrinsic and the coupling resonances.

Analogous to the method of using a vertical rf dipole at the intrinsic spin resonance, one should also expect to obtain a full spin flip by inducing a strong artificial resonance if the intrinsic and its coupling spin resonances are fully overlapped. Because of the coupling effect, the two spin resonances can never be brought closer than the minimum tune split $\Delta Q_{\min }$. However, $\Delta Q_{\min }$ in general is small and a full spin flip still should be achievable if the induced resonance is strong enough. In a fully coupled machine, the unperturbed tunes are equal and the intrinsic and the coupling resonances are equally strong and located on either side of the unperturbed betatron tune at a distance of half of $\Delta Q_{\min }$.

Unlike using a vertical rf dipole to obtain a vertical coherence in an uncoupled machine [10], the vertical coher- 
ence is excited by a horizontal rf dipole instead in a fully coupled machine. To understand this, let's first transform the beam motion from the normal geometric coordinates $(x, z, s)$ to the $(u, v, s)$ coordinate system in which the betatron motions along the two eigen directions are fully decoupled [6], i.e

$$
\left(\begin{array}{c}
x \\
x^{\prime} \\
z \\
z^{\prime}
\end{array}\right)=R\left(\begin{array}{c}
u \\
u^{\prime} \\
v \\
v^{\prime}
\end{array}\right) .
$$

where $R$ is the transformation matrix between $(x, z, s)$ and $(u, v, s)$. In a fully coupled machine with weak coupling coming from a solenoid magnet, namely the minimum tune split $\Delta Q_{\min }<<1$, the $R$ matrix is given by [11]

$$
R=\frac{1}{\sqrt{2}}\left(\begin{array}{cc}
I & A \\
A & I
\end{array}\right)
$$

with

$$
I=\frac{1}{\sqrt{2}}\left(\begin{array}{cc}
1 & 0 \\
0 & 1
\end{array}\right)
$$

and

$$
A=\frac{1}{\sqrt{2}}\left(\begin{array}{cc}
-a\left(\alpha_{x}+\alpha_{z}\right) & -a\left(\beta_{x}+\beta_{z}\right) \\
a\left(\gamma_{x}+\gamma_{z}\right) & a\left(\alpha_{x}+\alpha_{z}\right)
\end{array}\right),
$$

where $a=\frac{1}{\sqrt{\beta_{x} \gamma_{z}+\beta_{z} \gamma_{x}+2\left(1-\alpha_{x} \alpha_{z}\right)}}$ and $\beta_{x, z}$ are the horizontal and vertical betatron functions at where the solenoid magnet is. $\gamma_{x, z}=\frac{1+\alpha_{x, z}^{2}}{\beta_{x, z}}$ and $\alpha_{x, z}=-\frac{1}{2} \beta_{x, z}^{\prime}$ are the corresponding twiss parameters. Here ' is the derivative on the longitudinal coordinate $s$. With the weak coupling force, one also has [12]

$$
\begin{aligned}
\beta_{u} & \simeq \beta_{x} ; \quad \beta_{v} \simeq \beta_{z} \\
\alpha_{u} & \simeq \alpha_{x} ; \quad \alpha_{v} \simeq \alpha_{z}
\end{aligned}
$$

where $\beta_{u, v}$ and $\alpha_{u, v}$ are the twiss parameters in the two eigen directions.

With a horizontal rf dipole $\Delta B_{y} L=\Delta B_{y m} L \cos \nu_{m} \theta$, the horizontal excitation is $\delta x^{\prime} \cos \nu_{m} \theta=\frac{\Delta B_{y m} L}{B \rho} \cos \nu_{m} \theta$ where $B \rho$ is the magnetic rigidity. By transforming the fixed points in the $(u, v)$ coordinate system back to the normal geometric $(x, z)$ coordinates, one then obtains [11]

$$
\begin{array}{r}
f_{x} / b=0.0 \\
f_{x^{\prime}} / b=0.0 \\
f_{z} / b=a\left(\beta_{z} \alpha_{x}-\beta_{x} \alpha_{z}\right) \delta x^{\prime} \\
f_{z^{\prime}} / b=a\left(\beta_{x} \gamma z-\alpha_{x} \alpha_{z}+1\right) \delta x^{\prime}
\end{array}
$$

where $b=\frac{1}{4 \pi \delta}$. $\left(f_{x}, f_{x^{\prime}}\right)$ and $\left(f_{z}, f_{z^{\prime}}\right)$ are the horizontal and vertical fixed points in the normal geometric $(x, z)$ coordinates. This demonstrates that in a fully coupled machine, a vertical coherence can be excited by applying a horizontal rf dipole. The amplitude of the vertical coherence is $\frac{B L}{4 \pi B \rho \delta} \sqrt{\beta_{x} \beta_{z}}$.

Fig. 1 shows numerical spin tracking results at $G \gamma=$ $36+\nu_{z}$. The dotted line shows the result with the nominal AGS tune setting $\left(\nu_{x}=8.8, \nu_{z}=8.7\right)$ and no correction scheme for the intrinsic spin resonance. In this case, the depolarization at the coupling resonance is obvious. The solid line is the result of using a horizontal rf dipole with the horizontal and vertical betatron tunes set at 8.7. Due to the coupling from the solenoid partial snake, the two betatron tunes are split by 0.0144 . The horizontal rf dipole tune was set to 0.3 . With a horizontal rf dipole amplitude 28.0 G-m, a full spin flip was achieved.

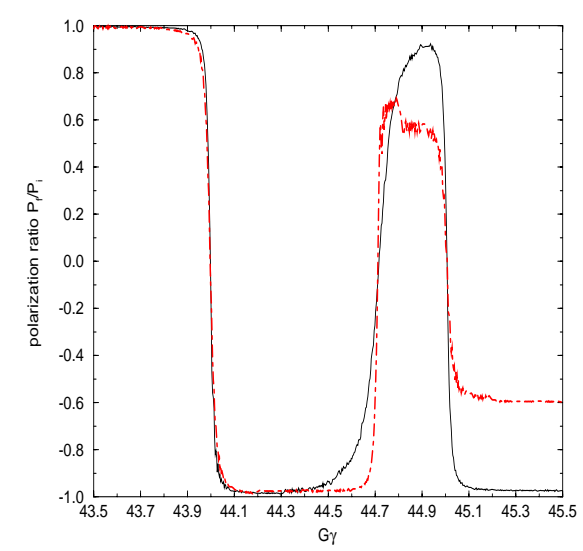

Figure 1: The two curves are the calculated polarization ratio $P_{f} / P_{i}$ as a function of energy. The solid line is for the case of a fully coupled machine and a horizontal rf dipole was used to obtain an adiabatic vertical coherence. The dotted line is the result of a weakly coupled machine with the two betatron tunes set 0.1 apart. No correction scheme was used at the $G \gamma=36+\nu_{z}$ intrinsic spin resonance. For both cases, the horizontal and vertical emittance are $20 \pi \mathrm{mm}$-mrad and $10 \pi \mathrm{mm}$-mrad respectively.

\section{EXPERIMENTAL RESULTS}

The method of using a horizontal rf dipole to excite a vertical coherence to cross the coupling spin resonance was tested in the AGS during the 2000 RHIC polarized proton commissioning run. The polarized $H^{-}$beam was preaccelerated in the $200 \mathrm{MeV}$ LINAC and then stripped and injected into the Booster. It was then injected into the AGS at $G \gamma=4.7$ and then accelerated up to $G \gamma=46.5$. In the AGS, the nominal tune setting is $\nu_{x}=8.8$ and $\nu_{z}=8.7$.

During the experiment, the AGS skew quadrupoles were all set to $17 \mathrm{~A}$. Due to a hardware limit, the partial snake strength at $G \gamma=36+\nu_{z}$ is actually only about $3.5 \%$ instead of 5\%. The combined effect of the skew quadrupoles and the weaker snake gave a smaller minimum tune spilt 
$\Delta Q_{\min }$ of 0.007 . The horizontal rf dipole was set in the middle of the two betatron tunes $\nu_{1}$ and $\nu_{2}$. The turn by turn beam position monitor data confirmed that a vertical coherence was excited without horizontal response as shown in the two left plots of Fig. 2. The horizontal response was not zero once the rf dipole tune deviated from the average of the two eigen tunes as shown on the right of Fig. 2.

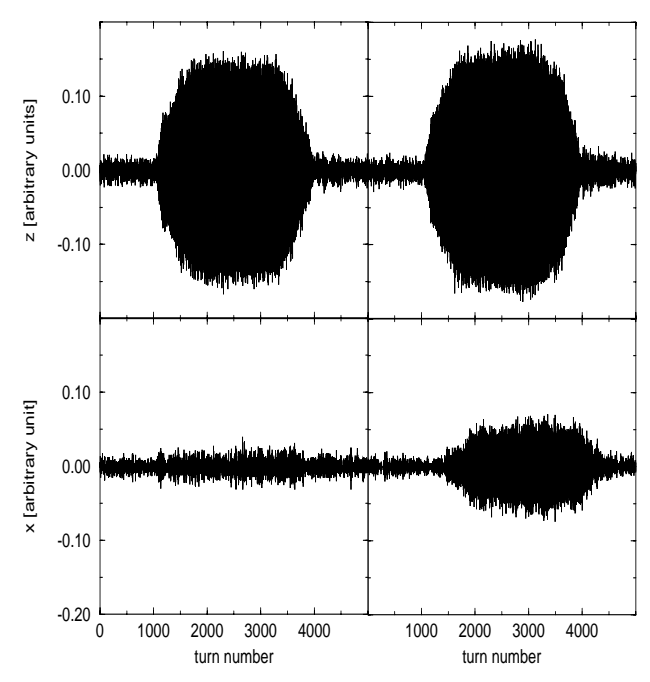

Figure 2: The top and bottom plots on the left are vertical and horizontal turn-by-turn beam position data when the horizontal rf dipole modulation tune $\nu_{m}=\frac{1}{2}\left(\nu_{1}+\nu_{2}\right)$. As shown, no horizontal coherence was excited. The two plots on the right correspond to the case where the horizontal modulation tune $\nu_{m} \neq \frac{1}{2}\left(\nu_{1}+\nu_{2}\right)$ and the horizontal coherence was no longer zero.

Table 1 shows the comparison of the measured beam asymmetries of using vertical $\mathrm{rf}$ dipole, no correction and using horizontal rf dipole at $G \gamma=36+\nu_{z}$. Comparing the measured asymmetry when using the horizontal $\mathrm{rf}$ dipole with the case of no correction, it is clear that the horizontal $\mathrm{rf}$ dipole did help to recover the beam polarization. However, the excited coherence was not optimized

Table 1: measured asymmetry

\begin{tabular}{|c|c|c|}
\hline & $\begin{array}{c}\text { measured asymmetry } \\
\left(\mathrm{x} 10^{-3}\right)\end{array}$ & condition \\
\hline 1 & $1.50 \pm 0.04$ & with vertical rf dipole \\
\hline 2 & $1.25 \pm 0.1$ & with horizontal rf dipole \\
\hline 3 & $0.067 \pm 0.063$ & no correction \\
\hline
\end{tabular}

and about $70 \%$ beam emittance growth was observed. Because of limitations of the AGS sextupole power supplies, we could not achieve small chromaticities in both planes and obtain a fully adiabatic excitation. This is the most likely reason that the horizontal rf dipole did not recover
$100 \%$ beam polarization as expected.

\section{CONCLUSION}

It has been demonstrated in the AGS that in a fully coupled machine, a vertical coherence can be excited by an horizontal rf dipole. Although beam polarization was improved, we think the residual polarization loss was due to the not fully adiabatic beam motion.

\section{ACKNOWLEDGEMENT}

We would like to thank Dr. L. Ahrens, Dr. E. D. Courant, W. J. Glenn, Dr. H. Huang, Dr. A. Lehrach, Dr. A. Luccio, Dr. W. Mackay, V. Ranjbar, Dr. N. Tsoupas, Dr. W. van Asselt for the fruitful discussions. We also would like to thank K. Zeno and D. Warburton for their great help. This work is performed under the auspices of Department of Energy of U.S.A.

\section{REFERENCES}

[1] S. Y. Lee, Accelerator Physics, World Scientific Pub. Singapore, 1999.

[2] D. A. Edwards, M. J. Syphers, An Introduction To The Physics of High Every Accelerators, Wiley-Interscience Pub. 1993.

[3] T. Roser, Partial Siberian Snake Test at the Brookhaven $A G S$, in High Energy Spin Physics: 10th International Symposium, ed. T.Hasegawa, et al., Nagoya, Japan, 1992, (Univesal Academic Press, Inc.,1992), p.429.

[4] E. D. Courant, R. D. Ruth, The Acceleration of Polarized Protons in Circular Accelerators, BNL report, BNL 51270, 1980.

[5] S. Y. Lee, Spin Dynamics and Snakes in Synchrotrons, World Scientific Pub. Singapore, 1997.

[6] D. A. Edwards, L. C. Teng, Parametrization of LINEAR Coupled Motion in Periodic Systems, IEEE Trans. on Nucl. Sc. 20, 885 (1973).

[7] H. Huang et al., Preservation of Proton Polarization by a Partial Siberian Snake, Phy. Rev. Letters. 73, 2982 (1994).

[8] H. Huang et al., Polarized Proton Beam in the AGS, Proceedings of $13^{\text {th }}$ international symposium in High Energy Spin Physics, P.492 (1998).

[9] M. Bai et al., Overcoming Intrinsic Spin Resonances with an rf Dipole, Physical Review Letters 80, 4673 (1998).

[10] M. Bai, et al., Experimental Test of Coherent Betatron Resonance Excitations, Physical Review E, 5(1997).

[11] M. Bai, T. Roser, Crossing a Coupling Spin Resonance with an RF Dipole, Brookhaven CAD/AP note C-A/AP/37 (2001).

[12] T. Roser, Multiturn Injection With Coupling, AGS/AD/Tech. Note No. 354. 